\title{
ON THE SPECTRA OF COMPACT NILMANIFOLDS
}

BY

JEFFREY S. FOX

\begin{abstract}
We show the equivalence of the Howe-Richardson multiplicity formula for compact nilmanifolds and the formula obtained by Corwin and Greenleaf using the Selberg trace formula.
\end{abstract}

Introduction. Let $G$ be a connected simply connected nilpotent Lie group and suppose $G$ contains a discrete cocompact subgroup $\Gamma$. Let $\rho=\operatorname{ind}_{\Gamma}^{G}(1)$. Then $\rho$ is a direct sum of irreducible representations each occurring with finite multiplicity; we will write $\rho=\oplus m(\pi) \pi$. A basic problem in representation theory is to determine $m(\pi)$ and give a criterion for $m(\pi)$ not to be zero. Moore first studied this problem in $[\mathbf{M}]$ and later Howe $[\mathbf{H}]$ and Richardson $[\mathbf{R}]$ independently gave a closed formula for $m(\pi)$ that generalized the classical Frobenius reciprocity formula for finite groups. Using the Poisson summation and Selberg trace formulas, Corwin and Greenleaf [C-G] gave a formula for $m(\pi)$ that depended only on the coadjoint orbit in $\mathrm{g}^{*}$ corresponding to $\pi$ via Kirillov theory and the structure of $\Gamma$, but the connection between the two formulas was not clear. In $\$ 1$ we consider the case when $\Gamma$ is a lattice subgroup of $G$, i.e. $\log (\Gamma)$ is an additive subgroup of the Lie algebra of $G$, and show there is a simple relationship between the two. In $\$ 2$ we show how Frobenius reciprocity can be used to reduce the general case to the lattice subgroup case.

Parts of this paper were in the author's doctoral dissertation and it is a pleasure to thank my advisor, Calvin Moore, for his support and guidance. I would also like to thank Larry Corwin for some useful conversation.

1. Let $G$ be a connected simply connected nilpotent Lie group. We denote the Lie algebra of $G$ by $g$ and the dual of $g$ by $g^{*}$. Let exp: $g \rightarrow G$ be the exponential map and $\log : G \rightarrow \mathfrak{g}$ its inverse. We let Ad be the adjoint action of $G$ on $g$ and $\mathrm{Ad}^{*}$ the coadjoint on $\mathrm{g}^{*}$. If $\pi$ is an irreducible unitary representation we write $\mathcal{O}(\pi) \subseteq \mathrm{g}^{*}$ for the coadjoint orbit associated to $\pi$ via Kirillov theory. Let $\Gamma \subseteq G$ be a discrete cocompact subgroup of $G$. If $Q$ denotes the rational numbers, then $\Gamma$ determines a $Q$ structure on $g$ by $\mathrm{g}_{Q}=\operatorname{span}_{Q}\{\log (\Gamma)\}$. We say $g \in G$ is rational iff $g=\exp (X)$ with $X \in \mathfrak{g}_{Q}$ and let $G_{Q}$ denote the set of rational points. Given a subalgebra $\mathfrak{h} \subseteq \mathfrak{g}$ we say $\mathfrak{h}$ is rational if $\mathfrak{h} \cap \mathfrak{g}_{Q}$ contains a basis of $\mathfrak{h}$ over $\mathbf{R}$. This is equivalent to $\exp (\mathfrak{h})=H$ having $H \cap \Gamma$ for a discrete cocompact subgroup. If $f \in \mathrm{g}^{*}$, say, $f$ is

Received by the editors May 30, 1984.

1980 Mathematics Subject Classification. Primary 22E25, Secondary 22E40, 22D30.

1985 American Mathematical Society $0002-9947 / 85 \$ 1.00+\$ .25$ per page 
rational if $f\left(\mathfrak{a}_{Q}\right) \subseteq Q$. For a very complete and detailed discussion of this see [C-G]. One should also note that if one views $G$ as an affine algebraic group, then the existence of a cocompact $\Gamma$ is equivalent to $G$ being defined over $Q$ and the notion of a rational point in the sense of algebraic groups is equivalent to the definition given above.

For the rest of this section we suppose that $\Lambda=\log (\Gamma)$ is an additive subgroup of g. Let $\Lambda^{\perp}=\left\{f \in \mathfrak{g}^{*} \mid f(\Lambda) \subseteq \mathbf{Z}\right\}$. Let $\pi$ be an irreducible representation of $G$ and $\mathcal{O} \subseteq \mathrm{g}^{*}$ the coadjoint orbit corresponding to $\pi$. According to [M], we have $m(\pi)>0$ if and only if $\mathcal{O} \cap \Lambda^{\perp} \neq \varnothing$, so we will suppose this intersection is nonempty. Since $\mathcal{O} \cap \Lambda^{\perp}$ is $\operatorname{Ad}^{*}(\Gamma)$ invariant we can write it as a union of $\operatorname{Ad}(\Gamma)$ orbits. To each such orbit $\Omega \subseteq \mathcal{O} \cap \Lambda^{\perp}$ one can associate a number $C(\Omega)$ as follows:

Let $f \in \Omega$ and $g(f)=\left\{X \in \mathfrak{g} \mid \operatorname{ad}^{*}(X) f=0\right\}$. Then $g(f)$ is a rational subalgebra, so the $\mathbf{Z}$-rank of $\mathfrak{g}(f) \cap \Lambda$ is equal to $\operatorname{dim}(\mathfrak{g}(f))$. Choose a $\mathbf{Z}$-basis $X_{1}, \ldots, X_{n}$ of $\Lambda$ (consequently it is an $\mathbf{R}$ basis for $g$ ) such that $X_{1}, \ldots, X_{s} \operatorname{span} g(f)$ over $\mathbf{R}$. Let $A_{f}$ be the matrix with entries $f\left(\left[X_{i}, X_{j}\right]\right), s<i, j \leqslant n$. Then $\left|\operatorname{det}\left(A_{f}\right)\right|$ is independent of the basis satisfying the above conditions and depends only on the $\Gamma$ orbit of $f$ in $\mathcal{O} \cap \Lambda^{\perp}$. Set $C(\Omega)=\left|\operatorname{det}\left(A_{f}\right)\right|^{-1 / 2}$. Then $C(\Omega)$ is a positive rational number. The multiplicity formula of Corwin and Greenleaf can be written as

$$
m(\pi)=\sum_{\Omega \in \mathcal{O} \cap \Lambda^{\perp} / \operatorname{Ad}^{*}(\Gamma)} C(\Omega) .
$$

For details see [C-G].

We now describe the Howe-Richardson formula for $m(\pi)$. If $m(\pi)>0$ there exists a rational element $f \in \mathcal{O}(\pi)$, fix such an element $f$. Let $\mathfrak{h} \subseteq \mathfrak{g}$ be a rational polarization for $f$ and $H=\exp (\mathfrak{h})$ the connected subgroup of $G$ with Lie algebra $\mathfrak{h}$. For $\mathfrak{h} \in H$ let $\chi_{f}(\mathfrak{h})=\exp (2 \pi i f(\log (\mathfrak{h})))$. Then $\chi_{f}$ is a character of $H$ and $\pi$ is equivalent to ind ${ }_{H}^{G}\left(\chi_{f}\right)$. Let $X=\left\{\left(\operatorname{Ad}(g) \mathfrak{h}, \operatorname{Ad}^{*}(g) f\right) \mid g \in G\right\}$ and define an equivalence relation on $X$ by $\left(\operatorname{Ad}(g) \mathfrak{h}, \operatorname{Ad}^{*}(g) f\right) \sim\left(\operatorname{Ad}\left(g_{1}\right) \mathfrak{h}, \operatorname{Ad}^{*}\left(g_{1}\right) f\right)$ iff $\operatorname{Ad}(g) \mathfrak{h}=$ $\operatorname{Ad}\left(g_{1}\right) \mathfrak{h}$ and $\left.\operatorname{Ad}^{*}(g) f\right|_{\operatorname{Ad}(g) \mathfrak{h}}=\left.\operatorname{Ad}^{*}\left(g_{1}\right) f\right|_{\operatorname{Ad}(g) \mathfrak{h}}$. Let $C(\mathfrak{h}, f)$ denote the set of equivalence classes. Define $L(\mathfrak{h}, f) \subseteq C(\mathfrak{h}, f)$ by $\left(\operatorname{Ad}(g) \mathfrak{h}, \operatorname{Ad}^{*}(g) f\right) \in L(\mathfrak{h}, f)$ iff $\operatorname{Ad}(g) \mathfrak{h}$ is a rational subalgebra of $g$ and $\left.\chi_{\mathrm{Ad}^{*}(g) f}\right|_{g H_{g^{-1}} \cap \Gamma} \equiv 1$. There is a natural action of $\Gamma$ on $L(\mathfrak{h}, f)$ by

$$
\gamma \cdot\left(\operatorname{Ad}(g) \mathfrak{h}, \operatorname{Ad}^{*}(g) f\right)=\left(\operatorname{Ad}(\gamma g) \mathfrak{h}, \operatorname{Ad}^{*}(\gamma g) f\right)
$$

and the number of $\Gamma$ orbits in $L(\mathfrak{h}, f)$ is the multiplicity $m(\pi)$. In what follows we will assume that $\mathfrak{h}$ and $f$ have been chosen such that $\mathfrak{h}$ is rational and $\left.\chi_{f}\right|_{H \cap \Gamma} \equiv 1$.

Suppose $\left.\chi_{\operatorname{Ad}^{*}(g) f}\right|_{g H^{-1} \cap \Gamma} \equiv 1$. Then $\operatorname{Ad}^{*}(g) f(\operatorname{Ad}(g) \mathfrak{h} \cap \Lambda) \subseteq \mathbf{Z}$ : thus we can find $\phi \in(\operatorname{Ad}(g) \mathfrak{h})^{\perp}$ such that $\operatorname{Ad}^{*}(g) f+\phi \in \Lambda^{\perp}$. From $\operatorname{Ad}^{*}\left(g \operatorname{Hg}^{-1}\right)\left(\operatorname{Ad}^{*}(g) f\right)$ $=\operatorname{Ad}^{*}(g) f+(\operatorname{Ad}(g) \mathfrak{h})^{\perp}$ we have $\operatorname{Ad}^{*}(g) f+\phi=\operatorname{Ad}^{*}(y)\left(\operatorname{Ad}^{*}(g) f\right)=\operatorname{Ad}^{*}(y g) f$ for some $y \in g \mathrm{Hg}^{-1}$. Since $\left(\operatorname{Ad}(g) \mathfrak{h}, \mathrm{Ad}^{*}(g) f\right)$ is equivalent to $\left(\operatorname{Ad}(y g) \mathfrak{h}, \operatorname{Ad}^{*}(y g) f\right)$ we have that every equivalence class in $L(\mathfrak{h}, f)$ has a representative $\left(\operatorname{Ad}(g) \mathfrak{h}, \operatorname{Ad}^{*}(g) f\right)$ such that $\operatorname{Ad}^{*}(g) f \in \Lambda^{\perp}$. We can now define a surjective map $\alpha$ : $\mathcal{O} \cap \Lambda^{\perp} \rightarrow L(\mathfrak{h}, f)$. If $\phi \in \Lambda^{\perp}$, then both $\phi$ and $f$ are rational 
points in $\mathcal{O}$; it follows [B or $\mathbf{M}$ ] that there exists $g \in G_{Q}$, the rational points of $G$, such that $\operatorname{Ad}^{*}(g) f=\phi$. Define $\alpha(\phi)=\alpha\left(\operatorname{Ad}^{*}(g) f\right)=\left(\operatorname{Ad}(g) \mathfrak{h}, \operatorname{Ad}^{*}(g) f\right)$. Since $g$ is rational, $\operatorname{Ad}(g) \mathfrak{h}$ is a rational subalgebra of $\mathfrak{g}$ and $\phi \in \Lambda^{\perp}$ automatically says $\left.\chi_{\operatorname{Ad}^{*}(g) f}\right|_{g H g^{-1} \cap \Gamma} \equiv 1$. Let $G(f)=\left\{g \in G \mid \operatorname{Ad}^{*}(g) f=f\right\}$; since $G(f) \subseteq H$ for any polarization $H$ we see that $\alpha$ is well defined. Since $\alpha$ is a $\Gamma$ equivariant map we can write the Corwin-Greenleaf formula as follows:

$$
m(\pi)=\sum_{\omega \in L(\mathfrak{h}, f) / \operatorname{Ad}^{*}(\Gamma)} \sum_{\Omega \in \alpha^{-1}(\omega)} C(\Omega) .
$$

The equivalence of the Howe-Richardson formula and the Corwin-Greenleaf formula for $\Gamma$ a lattice subgroup follows from above once we show

THEOREM 1. With notation as above $\sum_{\Omega \in \alpha^{-1}(\omega)} C(\Omega)=1$.

Before we begin with the proof of Theorem 1 we need the following lemmas from [H and $\mathbf{C - G}$.

Lemma 1. Let $\Gamma \subseteq G$ be a discrete cocompact subgroup of $G$, suppose $Z=$ center of $\mathrm{g}$ is one-dimensional and let $z \in \log (\Gamma) \cap Z$ be a generator. Then there exists $y \in \mathfrak{g}$ such that if $W=$ span of $y$ and $z$ over $\mathbf{R}$, then $y$ and $z$ generate $\log (\Gamma) \cap W$. Let $\mathfrak{g}_{1}=$ the centralizer of $y$ in $\mathfrak{g}$. Then $\mathfrak{g}_{1}$ is rational and of codimension 1 in $\mathfrak{g} . \mathfrak{g}_{1}$ is the Kirillov codimension 1 subalgebra $[\mathbf{K}]$. There is $x \in \log (\Gamma)$ such that if $\Gamma_{1}=\Gamma$ $\cap \exp \left(\mathrm{g}_{1}\right)$, then $\Gamma_{1}$ and $\exp (x)$ generate $\Gamma$. If $L=$ span over $\mathbf{R}$ of $x, y, z$, then $L$ is a three-dimensional Heisenberg algebra and $\exp (x), \exp (y), \exp (z)$ generate $\exp (L) \cap$ $\Gamma$. Finally, there exists $a \in \mathbf{Z}, a \neq 0$, so that $[x, y]=a z$ and $a$ is independent of the choice of $x$ satisfying the above conditions.

Definition 1. Let $\Gamma$ be a discrete torsion-free nilpotent group. A weak Malcev basis for $\Gamma$ is a set $\left\{d_{1}, \ldots, d_{p}\right\} \subseteq \Gamma$ such that:

(i) For any $d \in \Gamma$ there is a decomposition $d \equiv d_{1}^{n_{1}} \cdots d_{p}^{n_{p}}$, where $n_{i} \in \mathbf{Z}$.

(ii) The set $\Gamma_{i}=d_{1}^{\mathbf{Z}} \cdots d_{i}^{\mathbf{Z}}$ is a subgroup with $\Gamma_{i-1}$ normal in $\Gamma_{i}$ for $i=1,2, \ldots, p$.

(iii) $\Gamma_{i} / \Gamma_{i-1} \approx \mathbf{Z}$ for $i=2, \ldots, p$.

DefinITION 2. Let $G$ be a simply connected nilpotent Lie group. A weak Malcev basis for $G$ is a set $\left\{X_{1}, \ldots, X_{p}\right\} \subseteq \mathfrak{g}$ such that:

(i) For $X \in G \exists t_{i} \in \mathbf{R}, i-1, \ldots, p$, such that $X=\gamma_{1}\left(t_{1}\right) \cdots \gamma_{p}\left(t_{p}\right)$, where $\gamma_{i}(t)=$ $\exp \left(t X_{i}\right)$.

(ii) The set $G_{i}=\gamma_{1}(\mathbf{R}) \cdots \gamma_{i}(\mathbf{R})$ is a closed subgroup of $G$ with $G_{i-1}$ normal in $G_{i}$ for each $i$.

(iii) $G_{i} / G_{i-1} \approx \mathbf{R}$.

Weak Malcev basis is an adaptation of Malcev's coordinates of the 2 nd kind as necessitated by inducing from nonnormal subgroups in the Kirillov model [Ma].

If $\Gamma \subseteq G$ is a discrete cocompact subgroup of $G$ we say a weak Malcev basis $\left\{X_{1}, \ldots, X_{n}\right\}$ of $\mathrm{g}$ is subordinate to $\Gamma$ if $\left\{\exp \left(X_{1}\right), \ldots, \exp \left(X_{n}\right)\right\}$ is a weak Malcev basis for $\Gamma$. 
Lemma 2. Let $\Gamma$ be a discrete cocompact subgroup of $G$; if $\left\{d_{1}, \ldots, d_{n}\right\}$ is a weak Malcev basis for $\Gamma$, then $\left\{X_{i}=\log \left(d_{i}\right) \mid i=1, \ldots, n\right\}$ is a weak Malcev basis of $G$ subordinate to $\Gamma$.

Lemma 3. Let $G$ and $\Gamma$ be as above, $M \subseteq G$ a closed connected subgroup of $G$ such that $M / M \cap \Gamma$ is compact. If $\left\{X_{1}, \ldots, X_{s}\right\}$ is a weak Malcev basis of $M$ subordinate to $M \cap \Gamma$, then it can be extended to a weak Malcev basis of $G$ subordinate to $\Gamma_{0}$.

Lemma 4. If $\Gamma$ is a lattice subgroup of $G$ and $\left\{X_{1}, \ldots, X_{n}\right\}$ is a weak Malcev basis of $G$ subordinate to $\Gamma$, then $\left\{X_{1}, \ldots, X_{n}\right\}$ forms a $\mathbf{Z}$ basis of $A=\log (\Gamma)$.

Proof of Theorem 1. We proceed by induction on $\operatorname{dim}(G) . \operatorname{If} \operatorname{dim}(G)=1$ the statement is trivial and therefore suppose $\operatorname{dim}(G)>1$. Let $z$ be the center of $\mathrm{g}$; if $\operatorname{dim}(\operatorname{ker}(f) \cap z)>1$ we can divide out the corresponding central subgroup and proceed to a lower dimensional case. Consequently we can assume $\operatorname{dim}(z)=1$, and if $x, y, z$ and $g_{1}$ are as in Lemma 1 , then $f(z)=\lambda \neq 0$. Note that for $f_{1}, f_{2} \in \mathcal{O}(\pi)$, $f_{1}(z)=\operatorname{Ad}^{*}(g) f_{2}(z)=f_{2}(z)=\lambda$.

We can assume $\omega=\Gamma \cdot(\mathfrak{h}, f)$ simply by relabeling. To find $\Gamma$ orbits in $\mathcal{O} \cap \Lambda^{\perp}$ such that $\alpha(\Omega)=\omega$ we proceed as follows: The point $(H, f) \in \omega$ has $\Gamma \cap H$ for its stability group; consequently $\Gamma \cap H$ preserves the fiber $\alpha^{-1}((H, f))=\left(f+\mathfrak{h}^{\perp}\right) \cap$ $\Lambda^{\perp}$. A set of representatives for $\Gamma$ orbits in $\mathcal{O} \cap \Lambda^{\perp}$ that map into $\omega$ via $\alpha$ is given by a set of representatives of $\Gamma \cap H$ orbits in $\left(f+\mathfrak{h}^{\perp}\right) \cap \Lambda^{\perp}$.

As usual, there are two cases we must consider.

Case I. Suppose $\mathfrak{h} \subseteq \mathfrak{g}_{1}$. Let $\bar{f}$ be the restriction of $f$ to $\mathfrak{g}_{1}$. Then $\mathfrak{h}$ is a rational polarization for $\bar{f}$ and $\bar{\pi}=$ ind $_{H}^{G_{1}}\left(\chi_{\bar{f}}\right)$ occurs in $L^{2}\left(G_{1} / \Gamma_{1}\right)$ by the criterion in [C-G or M], i.e., $\bar{f} \in \Lambda_{1}^{\perp} \subseteq \mathfrak{g}_{1}^{*}$. Let $\mathfrak{h}^{\perp 1}=\left\{\phi \in \mathfrak{g}_{1}^{*} \mid \phi(x)=0 \forall X \in \mathfrak{h}\right\}$ and let $r: \mathfrak{g}^{*} \rightarrow \mathfrak{g}_{1}^{*}$ be the restriction map-so $r$ is Ad* equivariant. If $L=\left(f+\mathfrak{h}^{\perp}\right) \cap \Lambda^{\perp}$ and $L_{1}=(\vec{f}$ $\left.+h^{\perp 1}\right) \cap \Lambda_{1}^{\perp}$, then $\left.r\right|_{L}: L \rightarrow L_{1}$ is a $\Gamma \cap H=\Gamma_{1} \cap H$ equivariant surjective map. If $S_{1} \subseteq L_{1}$ is a set of $\Gamma \cap H$ orbit representatives, then $\left(\left.r\right|_{L}\right)^{-1}\left(S_{1}\right)$ contains a set of $\Gamma \cap H$ orbit representatives in $L$, say $S$. Let $\bar{\phi} \in S_{1}, \phi \in\left(\left.r\right|_{L}\right)^{-1}(\bar{\phi}), G_{1}(\phi)=$ stability group of $\bar{\phi}$ in $G$, and $G(\phi)$ be the stability group of $\phi$ in $G$. Then $G_{1}(\bar{\phi})=G(\phi)$. $\{\exp (t Y) \mid t \in \mathbf{R}\}$. Consequently a $\Gamma \cap G_{1}(\bar{\phi})$ orbit in $\left(\left.r\right|_{L}\right)^{-1}(\bar{\phi})$ is the same as a $\Gamma \cap\{\exp (t Y) \mid t \in \mathbf{R}\}=\{\exp (n Y) \mid n \in \mathbf{Z}\}$ orbit in $\left(\left.r\right|_{L}\right)^{-1}(\phi)$. An element $\phi \in$ $\left(\left.r\right|_{1}\right)^{-1}(\bar{\phi})$ is determined by its value on $x \in \mathfrak{g}$. Suppose $\phi(x)=b$. Then $\operatorname{Ad}^{*}(\exp (n Y))(\phi)(x)=b+n a \lambda$ and we see there are $|a \lambda|\{\exp (n Y) \mid n \in \mathbf{Z}\}$ orbits in $\left(\left.r\right|_{l}\right)^{-1}(\bar{\phi})$.

Let $\phi \in\left(\left.r\right|_{L}\right)^{-1}(\bar{\phi})$ for some $\bar{\phi} \in S_{1}$. We want to compute $C(\Gamma \cdot \phi)$. To do this we need a basis $X_{1}, \ldots, X_{n}$ of $\mathfrak{g}$ such that the $\mathbf{Z}$ span is $\Lambda$ and $X_{1}, \ldots, X_{s}$ span $\mathfrak{g}(\phi)$ over R. Using Lemmas 3 and 4 we can find a basis $X_{1}=z, X_{2}=y, \ldots, X_{n-1}$ of $\mathfrak{g}_{1}$ such that $X_{1}, \ldots, X_{n-1}$ span $\Lambda_{1}$ over $\mathbf{Z}, X_{1}, X_{2}, \ldots, X_{s}$ span $\mathfrak{g}_{1}(\bar{\phi})$, and $X_{1}, X_{3}, X_{4}, \ldots, X_{s}$ span $q(\phi) \subseteq \mathfrak{G}_{1}(\bar{\phi})$. We have $X_{1}, X_{3}, \ldots, X_{n-1} y, x$ span $\Lambda$ over $\mathbf{Z}$ and $X_{1}, X_{3}, \ldots, X_{s}$ span $g(\phi)$. Using this basis we can compute $C(\Gamma \circ \phi)=C(\phi)$. Recall that $C(\phi)=$ $|\operatorname{det}(A(\phi))|^{1 / 2}$, where $A(\phi)=\left(\phi\left(\left[x_{i}, x_{j}\right]\right)\right), s \leqslant i, j \leqslant n$. Since $\left[Y, \mathfrak{g}_{1}\right]=0$, if we expand $A(\phi)$ on that row and column, using $\phi([x, y])=\lambda a$, we get $\operatorname{det}(A(\phi))=$ $|\lambda a|^{2} \operatorname{det}(A(\bar{\phi}))$. Consequently, we have $C(\phi)=|\lambda a|^{-1} \cdot C(\bar{\phi})$ for all $\bar{\phi} \in S_{1}$. 
By our induction hypothesis $\sum_{\bar{\phi} \in S_{1}} C(\bar{\phi})=1$, so we get

$$
\begin{aligned}
\sum_{\phi \in S} C(\phi) & =\sum_{\bar{\phi} \in S_{1}}\left(\sum_{\phi \in(r \mid .)^{-1}(\bar{\phi}) \cap S} C(\phi)\right) \\
& =\sum_{\bar{\phi} \in S_{1}} C(\bar{\phi})\left(\sum_{\phi \in(r \mid 1 .)^{-1}(\bar{\phi}) \cap S}|\lambda a|^{-1}\right)=\sum_{\bar{\phi} \in S_{1}} C(\bar{\phi})=1 .
\end{aligned}
$$

Thus Case I is verified.

Case II. Now suppose $\mathfrak{h} \nsubseteq \mathfrak{g}_{1}$ and set $\mathfrak{h}_{0}=\mathfrak{h} \cap \mathfrak{g}_{1}, \overline{\mathfrak{h}}=\operatorname{span}\left(\mathfrak{h}_{0}, Y\right)$. Then $\overline{\mathfrak{h}} \subseteq \mathfrak{g}_{1}$ and is a polarization for $f$. As before, let $r: g^{*} \rightarrow g_{1}^{* \prime}$ be the restriction map. Then $\left.r\right|_{f+\mathfrak{h}^{\perp}}: f+\mathfrak{h}^{\perp} \rightarrow \mathfrak{g}_{1}^{*}$ is injective and $r\left(f+\mathfrak{h}^{\perp}\right)=\bigcup_{s \in \mathbf{R}}\left(f+s y^{*}+\overline{\mathfrak{h}}^{\perp 1}\right)$. Consequently we have $r\left(\left(f+\mathfrak{h}^{\perp}\right) \cap \Lambda^{\perp}\right)=\bigcup_{n \in \mathbf{Z}}\left(f+n y^{*}+\mathfrak{h}^{\perp 1}\right) \cap \Lambda_{1}^{\perp}$. A set $S$ of $\Gamma \cap H$ orbit representatives in $\left(f+\mathfrak{h}^{\perp}\right) \cap \Lambda^{\perp}$ can be written as $\bigcup_{0 \leqslant b<|\lambda a|} S_{b}$, where $S_{h}$, is a set of $\Gamma \cap \bar{H}$ orbit representatives in $\left(f+b y^{*}+\overline{\mathfrak{h}}^{\perp 1}\right) \cap \Lambda_{1}^{\perp}$ and $b \in \mathbf{Z}$. As before, if $\phi \in r^{-1}\left(S_{b}\right) \cap \Lambda^{\perp}$, then $C(\phi)=|\lambda a|^{-1} C(\bar{\phi})$, where $\bar{\phi}=r(\phi)$. Thus we have

$$
\begin{aligned}
\sum_{\phi \in S} C(\phi) & =\sum_{0 \leqslant h<|\lambda a|}\left(\sum_{\phi \in r^{-1}\left(S_{h}\right) \cap S} C(\phi)\right) \\
& =\sum_{0 \leqslant h<|\lambda a|}|\lambda a|^{-1}\left(\sum_{\bar{\phi} \in S_{b}} C(\bar{\phi})\right)=\sum_{0 \leqslant b<|\lambda c|}|\lambda a|^{-1}=1 . \quad \text { Q.E.D. }
\end{aligned}
$$

Corollary 1 (Moore $[\mathbf{M}]) \cdot m(\pi) \leqslant \#\left\{\mathcal{O} \cap \Lambda^{\perp} / \operatorname{Ad}^{*}(\Gamma)\right\}$.

Proof. By the above proof, $C(\phi)^{-1}=|\lambda a| C(\bar{\phi})$; thus we can reason by induction to conclude that $C(\phi)^{-1}$ is an integer. Q.E.D.

In [R] Richardson constructed a polarization for $f$ such that in the inductive reasoning one never has Case II occurring. We note that if $\mathfrak{h}$ is a Richardson polarization, then the above proof shows that the $C(\Omega)$ s are the same for every $\Omega$ such that $\alpha(\Omega)=\omega$. Since $\sum_{\alpha(\Omega)=\omega} C(\Omega)=1, C(\Omega)$ equals the number of $\Gamma \cap H$ orbits in $f+\mathfrak{h}^{\perp} \cap \Lambda^{\perp}$. This observation was pointed out to me by Larry Corwin.

2. If $\pi$ is a unitary representation of $G$ on the Hilbert space $H(\pi)$, we let $H^{\infty}(\pi)=\left\{u \in H(\pi) \mid q \rightarrow \pi(q) u\right.$ is a $C^{\infty}$-mapping $\}$. There is a representation of $g$, the Lie algebra of $G$, on $H^{\infty}(\pi)$. If $X \in \mathfrak{g}, u \in H^{\infty}(\pi)$ define

$$
\pi(X) u=\left.\frac{d}{d t}(\pi(\exp (t X) u))\right|_{t=0} .
$$

Then $X \rightarrow \pi(X)$ is a Lie algebra representation of $\mathfrak{g}$, so it extends to a representation of $\mathfrak{U}(\mathfrak{g})$. Given $a \in \mathfrak{U}(\mathfrak{g})$ define a seminorm on $H^{\infty}(\pi)$ by $\rho_{a}(u)=\|\pi(a) u\|$. Then $H^{\infty}(\pi)$ has the structure of a Fréchet space with respect to these seminorms and we let $H^{-\infty}(\pi)$ be the topological dual of $H^{\infty}(\pi)$. For details see [P-1]. We write $\pi^{\infty}$ for the restriction of $\pi$ to $H^{\infty}(\pi)$ and $\pi^{-\infty}$ for the dual representation of $G$ on $H^{-\infty}(\pi)$. The following is in [P-1]. 
TheOREM 2.1 (PENNEY). Let $G$ be a Lie group, $\Gamma$ a discrete cocompact subgroup and $\pi$ an irreducible representation of $G$. Then

$$
\operatorname{Hom}_{G}\left(\pi, \operatorname{ind}_{\Gamma}^{G}(1)\right) \simeq \operatorname{Hom}_{\Gamma}\left(1, \pi^{-\infty}\right) .
$$

If we set $\Gamma(\pi)=\left\{D \in H^{-\infty}(\pi) \mid \pi^{-\infty}(\lambda) D=D \forall \lambda \in \Gamma\right\}$, then the above theorem says $\operatorname{dim}\left(\operatorname{Hom}_{G}\left(\pi, \operatorname{ind}_{\Gamma}^{G}(1)\right)\right)=\operatorname{dim}(\Gamma(\pi))$.

When $G$ is a connected simply connected nilpotent Lie group, the lift maps of Richardson can be viewed as providing a basis of $\Gamma(\pi)[\mathbf{R}]$. If $\pi=\operatorname{ind}_{H}^{G}\left(\chi_{f}\right)$, then $H^{\infty}(\pi)$ corresponds to all Schwartz functions on $G / H[\mathbf{K}]$ and for each $\Gamma$ orbit in $L(\mathfrak{h}, f)$ one can construct an element of $\Gamma(\pi)$. If $\left(\operatorname{Ad}(g) \mathfrak{h}, \operatorname{Ad}^{*}(g) f\right)$ is a point of $L(\mathfrak{h}, f)$, then we can construct $D_{g} \in H^{-\infty}(\pi)$ as follows: For $\phi \in H^{\infty}(\pi)$ let $D_{g}(\phi)=\sum_{\gamma \in \Gamma / \Gamma \cap g H^{-1}} \phi(\gamma g)$. The $D_{g}$ 's are linearly independent for $g$ 's in different $\Gamma: H$ double cosets and they span $\Gamma(\pi)[\mathbf{P}-2, \mathbf{F}]$.

Now suppose $\Gamma_{0} \subseteq \Gamma$ is a normal subgroup of finite index and we know the truth of the Howe-Richardson formula for $\Gamma_{0}$.

Let $L_{0}(\mathfrak{h}, f)$ be defined using $\Gamma_{0}$ and $L(\mathfrak{h}, f)$ be defined using $\Gamma$. Of course $L(\mathfrak{h}, f) \subseteq L_{0}(\mathfrak{h}, f)$, so we will suppose $L_{0}(\mathfrak{h}, f)$ is not empty. Let $D_{g} \in \Gamma_{0}(\pi) \subseteq$ $H^{-\infty}(\pi)$. For $\gamma \in \Gamma, \phi \in H^{\infty}(\pi)$ we have

$$
\left(\pi^{-\infty}(\gamma) D_{g}\right)(\phi)=\sum_{\delta \in \Gamma_{0} / \Gamma_{0} \cap g H g^{-1}} \phi(\gamma \delta g)=\sum_{\delta \in \Gamma_{0} / \Gamma_{0} \cap \gamma g H g^{-1} \gamma^{-1}} \phi(\delta \gamma g) .
$$

Thus $\pi^{-\infty}(\gamma)$ stabilizes $\mathbf{C} \cdot D_{g}$ iff $\gamma \in \Gamma_{0} \cdot\left(\Gamma \cap g g^{-1}\right)$, then

$$
\pi^{-\infty}(\gamma) D_{g}=\chi_{f}\left(g^{-1} \gamma^{-1} g\right) D_{g}=\bar{\chi}_{g \cdot f}(\gamma) \cdot D_{g}
$$

(where $\bar{\chi}_{g \cdot f}$ extends to a character of $\Gamma_{0} \cdot\left(\Gamma \cap g \mathrm{Hg}^{-1}\right)$ by being trivial on $\Gamma_{0}$ ). If we set $W_{g}=\operatorname{span}\left\{\pi^{-\infty}(\gamma) D_{g} \mid \gamma \in \Gamma\right\}$, then we see that $\left.\pi^{-\infty}\right|_{\Gamma}$ acting on $W_{g}$ is exactly $\operatorname{ind}_{\Gamma_{0}}^{\Gamma} \cdot\left(\Gamma \cap g g^{-1}\right)\left(\bar{\chi}_{q \cdot f}\right)$. Let $S$ be a set of representatives for $\Gamma$ orbits in $L_{0}(\mathfrak{h}, f)$, so given $g \in S$ we get $\left(\operatorname{Ad}(g) \mathfrak{h}, \operatorname{Ad}^{*}(g) f\right)$ or equivalently a $D_{g} \in H^{-\infty}(\pi)$. From above we see that the representation $\left.\pi^{-\infty}\right|_{\Gamma}$ acting on $\Gamma_{0}(\pi)$ is

$$
\bigoplus_{g \in S} \operatorname{ind}_{\Gamma_{0} \cdot\left(\Gamma \cap g g^{-1}\right)}^{\Gamma}\left(\bar{\chi}_{g \cdot f}\right) \text {. }
$$

Since $\Gamma(\pi) \subseteq \Gamma_{0}(\pi)$, we have that

$$
\begin{aligned}
n n \operatorname{Hom}_{\Gamma}\left(1, \pi^{-\infty}\right) & \approx \bigoplus_{g \in S} \operatorname{Hom}_{\Gamma}\left(1, \operatorname{ind}_{\Gamma_{0} \cdot\left(\Gamma \cap g H g^{-1}\right)}^{\Gamma}\left(\bar{\chi}_{q \cdot f}\right)\right) \\
& \approx \bigoplus_{g \in S} \operatorname{Hom}_{\Gamma_{0} \cdot\left(\Gamma \cap g g^{-1}\right)}\left(1, \bar{\chi}_{g \cdot f}\right) .
\end{aligned}
$$

Thus

$$
\operatorname{dim}\left(\operatorname{Hom}_{\Gamma}\left(1, \operatorname{ind}_{\Gamma_{0} \cdot\left(\Gamma \cap g H^{-1}\right)}^{\Gamma}\left(\bar{\chi}_{g \cdot f}\right)\right)\right)= \begin{cases}1 & \text { if }\left.\chi_{g \cdot f}\right|_{g H g^{-1} \cap \Gamma} \equiv 1, \\ 0 & \text { otherwise. }\end{cases}
$$

Finally those $g \in S$ such that $\left.\chi_{g \cdot f}\right|_{g H g^{-1} \cap \Gamma} \equiv 1$ are parametrized by $L(\mathfrak{h}, f)$. Thus we have

Proposition. If $\Gamma_{0} \subseteq \Gamma$ is a normal subgroup and the Howe-Richardson formula for $\Gamma_{0}$ is known, then the Howe-Richardson formula for $\Gamma$ is true. 
RemarK. It is shown in [M or $\mathbf{C - G}$ ] that for a given $\Gamma$ it is always possible to find a normal $\Gamma_{0}$ such that $\Gamma_{0}$ is a lattice subgroup.

\section{REFERENCES}

[B] A. Borel, Linear algebraic groups, Benjamin, New York, 1969.

[C-G] L. Corwin and F. Greenleaf, Character formulas and spectra of compact nilmanifolds, J. Funct. Anal. 21 (1976), 123-154.

[F] J. Fox, Frobenius reciprocity and extensions of nilpotent Lie groups (preprint 1984).

[H] R. Howe, On Frobenius reciprocity for unipotent algebraic groups over Q, Amer. J. Math. 93 (1971), 163-172.

[K] A. A. Kirillov, Unitary representations of nilpotent Lie groups, Russian Math. Surveys 17 (1962), 53-104.

[Ma] A. Malcev, On a class of homogeneous spaces, Amer. Math. Soc. Transl., ser. 1, no. 39 (1951), 33 pp.

[M] C. C. Moore, Decomposition of unitary representations defined by discrete subgroups of nilpotent groups, Ann. of Math. (2) 82 (1965), 146-182.

[P-1] R. Penney, Abstract Plancherel theorems and a Frobenius reciprocity theorem, J. Funct. Anal. 18 (1975), 177-190.

[P-2] R. Penney, F. Greenleaf and L. Corwin, A general character formula for irreducible projections on $I^{2}{ }^{2}$ of a nilmanifold, Math. Ann. 225 (1977), 21-32.

[R] L. Richardson, Decomposition of the $L^{2}$ space of a general compact nilmanifold, Amer. J. Math. 93 (1971), 173-190.

Department of Mathematics, Purdue University, West Lafayette, Indiana 47907 